\title{
UNSUPERVISED SEABED SEGMENTATION OF SYNTHETIC APERTURE SONAR IMAGERY VIA WAVELET FEATURES AND SPECTRAL CLUSTERING
}

\author{
David P. Williams \\ NATO Undersea Research Centre, Viale San Bartolomeo 400, 19126 La Spezia (SP), Italy
}

\begin{abstract}
An unsupervised seabed segmentation algorithm for synthetic aperture sonar (SAS) imagery is proposed. Each $2 \mathrm{~m} \times 2 \mathrm{~m}$ area of seabed is treated as a unique data point. A set of features derived from the coefficients of a wavelet decomposition are extracted for each data point. Spectral clustering is then performed with this data, which assigns the data points to clusters. This clustering result is then used directly to effect a segmentation of the SAS image into different seabed types. Experimental results on four real, measured SAS images demonstrate the promise of the proposed approach. Importantly, accurate image segmentation results are achieved on the large, challenging images without the aid of any training data or parameter estimation.
\end{abstract}

Index Terms - Seabed segmentation, spectral clustering, synthetic aperture sonar, wavelet features, unsupervised learning

\section{INTRODUCTION}

Seabed segmentation is the process by which one segments (an image of) a typically large area of seabed into different regions based on the characteristics of the seabed. For example, an area may be segmented into flat seabed, rocky seabed, and seabed characterized by sand ripples. The need to perform this seabed classification in an automated manner with no human intervention is motivated by the desire to conduct fully autonomous mine countermeasures missions with an autonomous underwater vehicle.

An implicit assumption of most classification algorithms is that the underlying statistics that generated the training and testing data are the same. However, in real applications, this assumption is often violated, leading to what is known as, among other terms, covariate shift (e.g., [1]). For example, if one collects training data and learns a classifier from one site, but then attempts to classify testing data collected at a different location, a fundamental mismatch in the data can lead to poor classification performance. This scenario motivates the use of a purely unsupervised seabed segmentation algorithm, in which no training data is required.

Most previous seabed segmentation approaches - which have used simple side-scan imagery, as opposed to higher resolution synthetic aperture sonar (SAS) imagery — are either supervised approaches that require significant amounts of training data (e.g., [2, $3]$ ), or involve substantial parameter estimation and attempt to segment only between shadow and reverberation (e.g., [4,5]).

In this work, an unsupervised seabed segmentation algorithm is proposed for SAS imagery. The approach first employs a set of wavelet-based features that capture textural characteristics of an image. These features are subsequently exploited by a spectral clustering algorithm that effects a successful segmentation of the image into distinct seabed types. Attractively, no training data or parameter estimation is required.
The remainder of this paper is organized in the following manner. Features for the task of seabed segmentation are proposed in Sec. 2. The spectral clustering algorithm is described briefly in Sec. 3. Experimental results of unsupervised seabed segmentation on real, measured SAS imagery are shown in Sec. 4. A discussion of the proposed approach is given in Sec. 5, before concluding comments are made in Sec. 6.

\section{FEATURES FOR SEABED SEGMENTATION}

In this work, the "atomic" unit for seabed classification is assumed to be a $2 \mathrm{~m} \times 2 \mathrm{~m}$ area of seabed. That is, each $2 \mathrm{~m} \times 2 \mathrm{~m}$ area of seabed corresponds to one data point. This particular size was chosen as a compromise among several factors. The larger the area chosen, the more likely that a single data point will have the unfavorable property of containing multiple types of seabed. However, if the area is too small, the distinguishing characteristics of the seabed that indicate a certain seabed type may be lost.

\subsection{Wavelet features}

The proposed set of wavelet-based features consists of 16 features that are derived from the coefficients of a biorthogonal wavelet decomposition [6] of each SAS image block (i.e., data point).

A wavelet decomposition of an image results in a set of decomposition coefficients that are computed via a cascade of filtering and subsampling. Each wavelet coefficient corresponds to a unique orientation and scale pair. The coefficients associated with one such pair comprise a "sub-image." In this work, we employ a compactly supported biorthogonal spline wavelet and perform a five-scale decomposition, which results in 16 such sub-images. The features that are used for the seabed segmentation correspond to the quadratic mean (i.e., root-mean-square (RMS) value) of the wavelet coefficient amplitudes of each sub-image.

This particular set of features was chosen because it can successfully capture the distinguishing textural properties of the seabed. Namely, the wavelet-coefficient energy will be large when the orientation and scale match the orientation and scale of high-energy texture components in an image block (i.e., data point) [6].

\subsection{Moment features}

A second, alternative set of four features we devise (for comparison purposes) are moment-based. Specifically, the features are the mean, variance, skewness, and kurtosis of the distribution of pixel values of an image block (i.e., data point). These features are motivated by the fundamental sonar scattering physics of the seabed. (For example, the amount of acoustic energy scattered back to the sonar receiver from areas of seabed characterized by sand ripples or rocks is larger than the amount scattered from flat, benign seabed. This and other 
similar insight suggest that these features would be able to discriminate among different seabed types.)

\section{SPECTRAL CLUSTERING}

Spectral clustering $[7,8]$ is a method that exploits the eigenvectors of a matrix composed of distances between data points to perform clustering. The advantage of spectral clustering lies in the fact that the natural clusters in the original feature space do not necessarily correspond to convex regions. The algorithm is described here by following the approach of [8].

Let $\boldsymbol{x}_{i} \in \mathbb{R}^{d}$ denote a vector of $d$ features representing the $i$ th data point. Consider a data set of $n$ data points, $\left\{\boldsymbol{x}_{i}\right\}_{i=1}^{n}$, that we wish to cluster into $k$ clusters.

Define the affinity matrix $\mathbf{A} \in \mathbb{R}^{n \times n}$ that expresses the similarity between each pair of data points as

$$
A_{i j}= \begin{cases}\exp \left\{-\left\|\boldsymbol{x}_{i}-\boldsymbol{x}_{j}\right\|^{2} / \sigma\right\}, & \text { if } i \neq j ; \\ 0, & \text { if } i=j,\end{cases}
$$

where $\sigma$ is a constant. Define the diagonal matrix $\mathbf{D}$ as $D_{i i}=$ $\sum_{j=1}^{n} A_{i j}$, and form the matrix $\mathbf{L}=\mathbf{D}^{-1 / 2} \mathbf{A D}^{-1 / 2}$.

Find the $m$ eigenvectors $-\boldsymbol{e}_{1}, \boldsymbol{e}_{2}, \cdots, \boldsymbol{e}_{m}-$ of $\mathbf{L}$ associated with the $m$ largest eigenvalues, and stack them in columns to form the matrix $\mathbf{E}=\left[\boldsymbol{e}_{1} \boldsymbol{e}_{2} \cdots \boldsymbol{e}_{m}\right] \in \mathbb{R}^{n \times m}$. Next construct the matrix $\mathbf{Z} \in \mathbb{R}^{n \times m}$ to be a re-normalized version of $\mathbf{E}$ whose rows have unit length, so $Z_{i j}=E_{i j}\left[\sum_{j=1}^{m} E_{i j}^{2}\right]^{-1 / 2}$.

Treat each row of $\mathbf{Z}$ as a data point in $\mathbb{R}^{m}$, meaning the original feature vector $\boldsymbol{x}_{i} \in \mathbb{R}^{d}$ has effectively been transformed into a new vector $\boldsymbol{z}_{i} \in \mathbb{R}^{m}$. Cluster the new data set, $\left\{\boldsymbol{z}_{i}\right\}_{i=1}^{n}$, into $k$ clusters via $k$-means.

The cluster assignment of the original data point $\boldsymbol{x}_{i}$ is exactly the cluster assignment of $\boldsymbol{z}_{i}$.

\section{EXPERIMENTS}

\subsection{Data set}

In April-May 2008, the NATO Undersea Research Centre (NURC) conducted the Colossus II sea trial in the Baltic Sea off the coast of Latvia. During this trial, high-resolution sonar data was collected by the MUSCLE autonomous underwater vehicle (AUV). This AUV is equipped with a $300 \mathrm{kHz}$ sonar with a $60 \mathrm{kHz}$ bandwidth that can achieve image resolution of approximately $3 \mathrm{~cm}$. The sonar data was subsequently processed into synthetic aperture sonar (SAS) imagery. We perform unsupervised seabed segmentation on four images from this data set. The first image spans an area of $56 \mathrm{~m} \times 56 \mathrm{~m}$ of seabed, while each of the other three images span an area of $50 \mathrm{~m} \times 110 \mathrm{~m}$.

To allow a quantitative assessment of the segmentation results, we manually ground truth the first SAS image, shown in Fig. 1(a), into three seabed types (namely, flat, rippled, and rocky seabed). The result of this ground truthing is shown in Fig. 1(b).

\subsection{Experimental set-up}

Each data point is taken to correspond to a unique $2 \mathrm{~m} \times 2 \mathrm{~m}$ area of seabed. The $d=16$ wavelet features and the $d=4$ moment features described in Sec. 2.1 and Sec. 2.2, respectively, are extracted for each data point.

To evaluate the utility of the wavelet features, we also conduct experiments that instead employ the moment features described in Sec. 2.2. To evaluate the utility of spectral clustering, we also conduct experiments that instead perform clustering using $k$-means (without first performing spectral clustering). To these ends, we consider four different unsupervised segmentation approaches, which differ both in the set of features employed and in the clustering method used. Specifically, segmentation is performed when using (i) moment features with $k$-means clustering, (ii) moment features with spectral clustering, (iii) wavelet features with $k$-means clustering, and (iv) wavelet features with spectral clustering (i.e., the proposed approach).

In all four cases, the segmentation process is completely unsupervised, so no training data is needed or used. For the cases that employ spectral clustering, only the top two eigenvectors $(m=2)$ are retained, meaning each data point to be clustered is $\boldsymbol{z}_{i} \in \mathbb{R}^{2}$. In (1), the value of $\sigma$ is set to $\sigma=\max _{i, j}\left\{\left\|\boldsymbol{x}_{i}-\boldsymbol{x}_{j}\right\|^{2} / 10\right\}$.

For all experiments reported here, the number of clusters to be learned is fixed at $k=4$. (We set $k=4$ rather than $k=3$ because the flat seabed type is often composed of two disparate subtypes, differentiated by whether shell deposits are present or absent.) Because the $k$-means algorithm is not guaranteed to result in the globally optimal clustering, 100 random cluster-centroid initializations are considered for each case. The clustering for which the distortion - defined as the sum of distances from each point to its assigned cluster centroid - is a minimum is selected as the final clustering (and by extension, the final segmentation).

\subsection{Results}

The results of the unsupervised seabed segmentation on the SAS image shown in Fig. 1(a) are shown visually in Figs. 1(c)-1(f), and in the form of confusion matrices (since the image was manually ground truthed) in Tables 1-4.

Because the methods are unsupervised, no explicit correspondence between clusters and seabed types exists. However, for purposes of evaluating the segmentation results here, one can easily assign a correspondence between ground-truth seabed types and clusters. In Tables 1-4, entries in bold are considered to be correct segmentations, with these quantities used to calculate the overall correct "classification" rates of each method, shown in Table 5.

As can be observed from Figs. 1(c)-1(f), Tables 1-4, and Table 5, the approach employing wavelet features with spectral clustering results in the best segmentation for the first test image.

We also apply the four methods to three additional SAS images, shown in Figs 2(a), 2(b), and 2(c). Due to space constraints, the segmentation results of only the proposed approach employing wavelet features and spectral clustering are shown, in Figs 2(d), 2(e), and 2(f), respectively. However, the general trend of the segmentation results (not shown) of the other three methods on these three images is similar to the results on the first image; that is, these alternative methods result in inferior segmentations.

\section{DISCUSSION}

Despite the justified use of the moment features, it was observed (in the results shown and others not shown) that the wavelet features were far superior to discriminating among different seabed types. It was also observed that performing spectral clustering prior to effecting a segmentation via $k$-means greatly improved the segmentation result when employing wavelet features. In the results not shown due to space constraints, applying $k$-means without spectral clustering resulted in significantly less appealing segmentations.

The main drawback of $k$-means - that the number of clusters to be found must be chosen a priori - is well-known and has been 
Table 1. Confusion matrix of the segmentation result using moment features and $k$-means ( $c f$. Fig. 1(d)). Entries in bold are treated as correct classifications.

\begin{tabular}{|c||c|c|c|c|}
\hline \multicolumn{1}{|c||}{} & \multicolumn{4}{c|}{ CLUSTER NUMBER } \\
GRound TRUTH & 1 & 2 & 3 & 4 \\
\hline \hline FLAT SEABED & $\mathbf{3 0 3}$ & $\mathbf{2 8}$ & 201 & 15 \\
\hline RIPPLED SEABED & 21 & 1 & $\mathbf{1 0 9}$ & 26 \\
\hline ROCKY SEABED & 18 & 9 & 16 & $\mathbf{3 7}$ \\
\hline
\end{tabular}

Table 2. Confusion matrix of the segmentation result using moment features and spectral clustering ( $c f$. Fig. 1(e)). Entries in bold are treated as correct classifications.

\begin{tabular}{|c||c|c|c|c|}
\hline \multicolumn{1}{|c||}{} & \multicolumn{4}{c|}{ CLUSTER NUMBER } \\
GROUND TRUTH & 1 & 2 & 3 & 4 \\
\hline \hline FLAT SEABED & $\mathbf{2 4 8}$ & $\mathbf{1 2 9}$ & 120 & 50 \\
\hline RIPPLED SEABED & 12 & 18 & $\mathbf{6 1}$ & 66 \\
\hline ROCKY SEABED & 10 & 20 & 13 & $\mathbf{3 7}$ \\
\hline
\end{tabular}

Table 3. Confusion matrix of the segmentation result using wavelet features and $k$-means ( $c f$. Fig. 1(f)). Entries in bold are treated as correct classifications.

\begin{tabular}{|c||c|c|c|c|}
\hline \multicolumn{1}{|c||}{} & \multicolumn{4}{c|}{ CLUSTER NUMBER } \\
GRound TRUth & 1 & 2 & 3 & 4 \\
\hline \hline FLAT SEABED & $\mathbf{2 8 6}$ & $\mathbf{1 2 8}$ & 10 & 123 \\
\hline RIPPLED SEABED & 0 & 4 & $\mathbf{1 5 0}$ & 3 \\
\hline ROCKY SEABED & 1 & 15 & 30 & $\mathbf{3 4}$ \\
\hline
\end{tabular}

Table 4. Confusion matrix of the segmentation result using wavelet features and spectral clustering ( $c f$. Fig. 1(c)). Entries in bold are treated as correct classifications.

\begin{tabular}{|c||c|c|c|c|}
\hline \multicolumn{1}{|c||}{} & \multicolumn{4}{c|}{ CLUSTER NUMBER } \\
Ground TRUth & 1 & 2 & 3 & 4 \\
\hline \hline FLAT SEABED & $\mathbf{3 4 9}$ & $\mathbf{1 4 3}$ & 9 & 46 \\
\hline RIPPLED SEABED & 0 & 2 & $\mathbf{1 2 8}$ & 27 \\
\hline ROCKY SEABED & 2 & 18 & 14 & $\mathbf{4 6}$ \\
\hline
\end{tabular}

Table 5. Correct classification rates for each method applied to the image in Fig. 1(a).

\begin{tabular}{|c||c|c|}
\hline \multicolumn{1}{|c||}{} & \multicolumn{2}{c|}{ Clustering METHOD } \\
FEATURES & $k$-MEANS & $\begin{array}{c}\text { SPECTRAL } \\
\text { CLUSTERING }\end{array}$ \\
\hline \hline MOMENTS & 0.6084 & 0.6059 \\
\hline WAVELETS & 0.7628 & $\mathbf{0 . 8 4 9 5}$ \\
\hline
\end{tabular}

addressed by various approaches (e.g., [9]). However, spectral clustering is not dependent on $k$-means; rather, any reasonable clustering method can be employed in the final step of spectral clustering. Our intent here was to illustrate only the added benefit of employing spectral clustering before performing the final clustering, so no attempt has been made to address issues inherent to $k$ means. If desired, one could instead employ an alternative final clustering method, such as a variational Bayesian Gaussian mixture model [10], which can automatically infer the appropriate number of mixtures (i.e., clusters) represented in the data.

\section{CONCLUSIONS}

A new, purely unsupervised approach based on wavelet features and spectral clustering was proposed for the task of seabed segmentation in synthetic aperture sonar (SAS) imagery. Promising segmentation results were obtained on challenging, real SAS imagery, despite the fact that no training data was used. Moreover, no parameter estimation was required.

Future work will consider the use of alternative clustering methods (instead of $k$-means) in the final step of the spectral clustering algorithm.

\section{REFERENCES}

[1] M. Sugiyama, M. Krauledat, and K. Müller, "Covariate shift adaptation by importance weighted cross validation," J. Mach. Learn. Res., vol. 8, pp. 985-1005, 2007.

[2] D. Carmichael, L. Linnett, S. Clarke, and B. Calder, "Seabed classification through multifractal analysis of sidescan sonar imagery," IEE Radar, Sonar and Navigation, vol. 143, no. 3, pp. 140-148, 1996.

[3] S. Reed, I. Ruiz, C. Capus, and Y. Petillot, "The fusion of large scale classified sidescan sonar image mosaics," IEEE Trans. Image Processing, vol. 15, no. 7, pp. 2049-2060, 2006.

[4] M. Mignotte, C. Collet, and P. Bouthemy, "Sonar image segmentation using an unsupervised hierarchical MRF model," IEEE Trans. Image Processing, vol. 9, pp. 1216-1231, 2000.

[5] K. Yao, M. Mignotte, C. Collet, P. Galerne, and G. Burel, "Unsupervised segmentation using a self-organizing map and a noise model estimation in sonar imagery," Pattern Recognition, vol. 33, pp. 1575-1584, 2000.

[6] S. Mallat, A Wavelet Tour of Signal Processing. Academic Press, 1999.

[7] M. Meilă and J. Shi, "Learning segmentation by random walks," in Advances in Neural Information Processing Systems 13. MIT Press, 2000, pp. 873-879.

[8] A. Ng, M. Jordan, and Y. Weiss, "On spectral clustering: Analysis and an algorithm," in Advances in Neural Information Processing Systems 14. MIT Press, 2001, pp. 849-856.

[9] R. Tibshirani, G. Walther, and T. Hastie, "Estimating the number of clusters in a dataset via the gap statistic," J. Royal Statist. Soc. B, vol. 63, pp. 411-423, 2001.

[10] M. Beal and Z. Ghahramani, "The variational Bayesian EM algorithm for incomplete data: Application to scoring graphical model structures," Bayesian Statistics, vol. 7, pp. 453-464, 2003. 


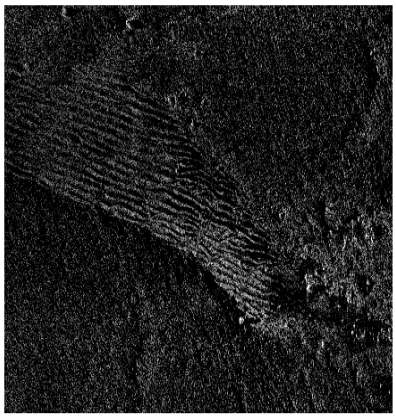

(a) SAS image



(d) Alternative segmentation result

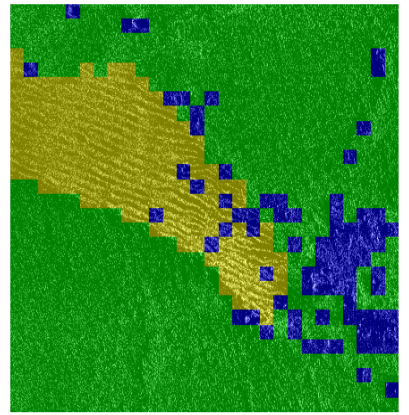

(b) Ground truth

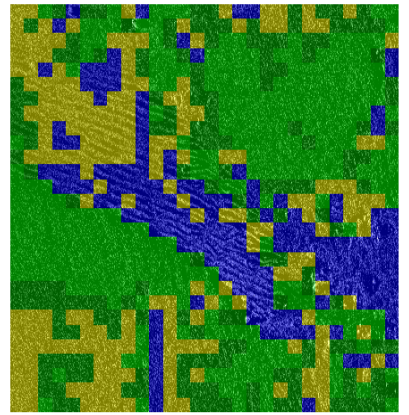

(e) Alternative segmentation result

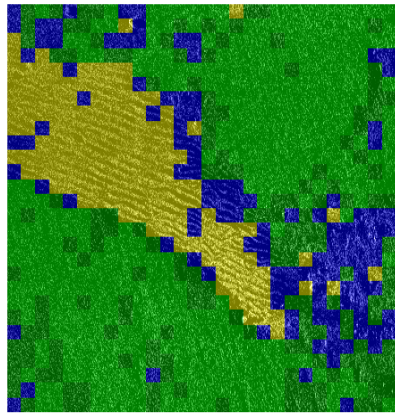

(c) Segmentation result (proposed)

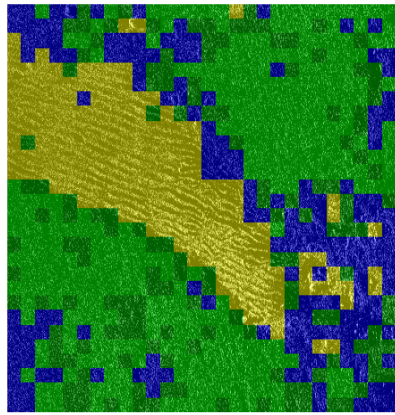

(f) Alternative segmentation result

Fig. 1. Segmentation results of the $56 \mathrm{~m} \times 56 \mathrm{~m} \mathrm{SAS}$ image are shown for the cases of using (c) wavelet features with spectral clustering (i.e., the proposed method), (d) moment features with $k$-means, (e) moment features with spectral clustering, and (f) wavelet features with $k$-means.

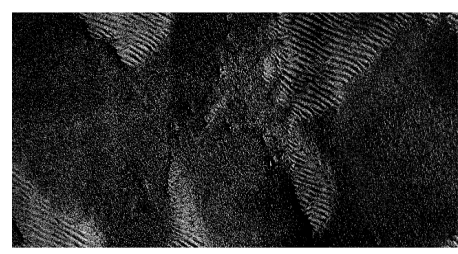

(a) SAS image

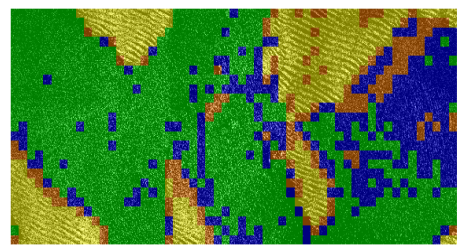

(d) Segmentation result

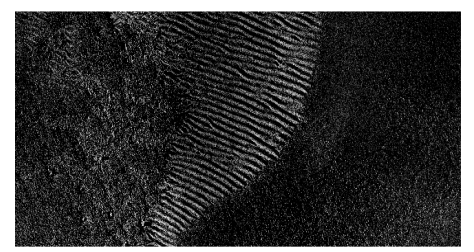

(b) SAS image

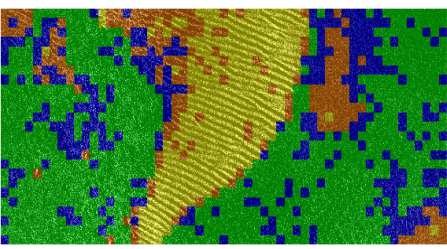

(e) Segmentation result

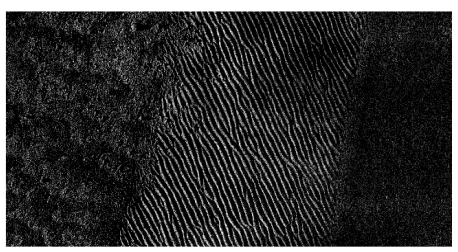

(c) SAS image

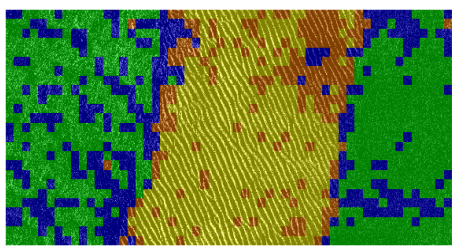

(f) Segmentation result

Fig. 2. Three additional SAS images, each covering an area of $50 \mathrm{~m} \times 110 \mathrm{~m}$, along with the corresponding unsupervised segmentation results from using wavelet features with spectral clustering. (N.B. In the electronic version of the paper, finer details of the SAS images can be observed when zooming in.) 